\title{
Die Meta-Basalte der Iberger Klippen
}

\section{Journal Article}

\section{Author(s):}

Dietrich, Volker J.

Publication date:

2006-05

Permanent link:

https://doi.org/10.3929/ethz-b-000001392

Rights / license:

In Copyright - Non-Commercial Use Permitted

\section{Originally published in:}

Eclogae Geologicae Helvetiae 99(1), https://doi.org/10.1007/s00015-006-1182-0 


\title{
Die Meta-Basalte der Iberger Klippen
}

\author{
VOLKER J. DIETRICH ${ }^{1}$
}

Key words: Alpine ophiolites, meta-basalts, Iberg Klippen nappe, Platta nappe, Arosa zone

\section{ZUSAMMENFASSUNG}

Die Meta-Basalte liegen als Meter mächtige tektonische Schuppen und Linsen in den Flyschen der Iberger Klippen und sind aufgrund der schlechten Aufschlussverhältnisse nur in Anschnitten von Waldstrassen aufgeschlossen (siehe Trümpy 2006, dieser Band). Meist handelt es sich einerseits um kataklastisch beanspruchte Pillowlaven, Pillowbrekzien und Hyaloklastite, andererseits um feinkörnige und grobkörnigere Diabase und Diabasbrekzien. Stellenweise sind diese Vulkanite jedoch stark tektonisiert und bis zur Unkenntlichkeit mylonitisiert.

Für alle Iberger Meta-Basalte kann die Klassifikation basaltischer Vulkanite (Pillowlaven, Pillowbrekzien, Hyaloklastite sowie Diabas Lagergänge) verwendet werden. Es fand lediglich eine schwache metamorphe Überprägung in tieferer Grünschieferfazies statt; primäre Strukturen wurden weitgehend erhalten. Generell können beide Gruppen von Iberger Vulkaniten (Pillows und Diabas-Gänge) als Basalte eines ehemaligen ozeanischen Rücken (analog «MORB» Middle Oceanic Ridge Basalt) gedeutet werden. Sie zeigen den typischen tholeiithischen Differentiationstrend, wobei die Diabase einen primitiven (undifferenzierten) olivin-basaltischen Charakter aufweisen.

Die Iberger Meta-Basalte, zusammen mit ihrer mittel- bis oberjurassischen ozeanischen Sedimentbedeckung (Radiolarit und Aptychenkalk), können mit der Arosa Zone, respektive den «Oberen Platta-Schuppen», parallelisiert werden. Sie sind Teile ozeanischer Kruste eines Piemont-Liguria Meeresbeckens mit gestaffelten mittelozeanischen Rücken und transformen Bruchsystemen, welches sich zwischen der Eurasischen Platte und dem paläogeographisch «südlichen» Kontinentalrand (Mosaik der Südalpinen, Adriatischen, respektive Ostalpinen Mikroplatten) erstreckte.

\section{ABSTRACT}

The meta-basalts are part of a typical ophiolite assemblage and occur as exotic thrust slices of several meters thickness within of the nappe pile of the «Iberg Klippen» (for a detailed description see Trümpy 2006, this volume). The outcrop conditions are very poor due to forest and dense vegetation. The volcanics consist of pillow lava, pillow breccia, hyaloclastite, as well as of compact diabase sills interlayered with the extrusive pillow sequences. We use the classification scheme for volcanites in case of these slightly metamorphosed basalts since all structures and micro-fabrics are mostly still well preserved.

All rock types (pillow lava, -breccia, hyaloclastite and diabase-sills) have been transformed to lower greenschist facies assemblages (pumpellyite-zone; Dietrich et al., 1974). Analcime may still be present according to an X-ray spectrum obtained on one sample. The mineral composition of the pillow lava consists of chlorite (ripidolite), sericite (muscovite), pumpellyite, sphene, hematite, albite, calcite and quartz. A high proportion of hematite and muscovite is present in reddish pillow lava and breccia. Meta-basaltic sills contain the minerals chlorite (ripidolite), albite, pumpellyite, sphene and calcite.

Generally, all Iberg volcanics may be interpreted as basalts derived from a mid-oceanic ridge (analogous to «MORB» Middle Oceanic Ridge Basalt). They show a typical tholeiitic differentiation trend, whereas diabase, in particular sample $\mathrm{Ob} 5$ (table 1) exhibits a quite undifferentiated olivine-basaltic character: $\mathrm{MgO}=11.68$ wt. $\%, \mathrm{Ni}=221 \mathrm{ppm}$ and $\mathrm{Cr}=426 \mathrm{ppm}$, as well as low $\mathrm{Ti}, \mathrm{Zr}, \mathrm{Y}, \mathrm{V}, \mathrm{Sc}$, low $\mathrm{K}_{2} \mathrm{O}$ and $\mathrm{Rb}$. In contrast, the hematitic pillow lava is fairly differentiated: $\mathrm{MgO}$ varies between 6.7 and 3.5 wt. \%, Cr between 277-264 ppm, Ni between 134-82 ppm, and higher concentrations in $\mathrm{Fe}, \mathrm{Ti}, \mathrm{Zr}, \mathrm{Y}, \mathrm{V}$, and Sc. The unusual high and variable concentrations of $\mathrm{Na}_{2} \mathrm{O}, \mathrm{K}_{2} \mathrm{O}$ and $\mathrm{Rb}$ are interpreted to have resulted from oceanic alteration and hydrothermal processes as well as from weak regional Alpine metamorphism. However, despite postmagmatic metasomatic effects, the basaltic composition exhibits primary characteristics of slightly enriched (transitional) MORB.

All data on the Iberg meta-basalts, including their stratigraphical contacts with the overlying upper Jurassic radiolarian cherts and calcareous limestones («Aptychenkalke», respectively «Calpionella limestones») and their chemical composition, indicate a close relationship to the «Arosa zone» and the «Upper Platta nappe thrust slices» of the southern Oberhalbstein valley (Grisons), Dietrich, 1969. Therefore it can be assumed, that these ophiolitic volcanics were part of an oceanic crust, cut by numerous transform faults and part of a rather small «Piemont ocean», representing the northern extension of the «Ligurian Tethys» located between the Eurasian plate and a «Southern» continental margin consisting of the South-Alpine, Adriatic and Austroalpine micro-plates.

\footnotetext{
${ }^{1}$ Institut für Mineralogie und Petrographie, ETH Zentrum, CH-8092 Zürich, Switzerland
} 


\section{Die Vorkommen}

Sämtliche Meta-Basalte liegen als Meter mächtige tektonische Schuppen und Linsen in den Flyschen der Iberger Klippen und sind aufgrund der schlechten Aufschlussverhältnisse nur in den Anschnitten der Waldstrassen aufgeschlossen (Trümpy 2006; dieser Band). Meist handelt es sich um kataklastisch beanspruchte Pillowlaven, Pillowbrekzien und Hyaloklastite, sowie um feinkörnige und grobkörnigere Diabase und Diabasbrekzien. Stellenweise sind jedoch diese Vulkanite stark tektonisiert und bis zur Unkenntlichkeit mylonitisiert.

Der grösste zusammenhängende Aufschluss im Schneiterwald (Koord. ca. 700.500 / 208.700 / 1320-1367 m.ü.M.) besteht fast durchwegs aus ca. $60 \mathrm{~cm}$ grossen, gegen NW einfallenden, normal gelagerten Pillows, welche von geringmächtigen roten Radiolariten bedeckt sind. Im oberen Teil, nahe am Kontakt zum Radiolarit, erscheinen die Pillowlaven deutlich grobkörniger, mit bis zu $6 \mathrm{~mm}$ langen grünlichweissen Plagioklasleisten.

Westlich des Bueffengadens (P. 1334), etwa auf der gleichen Höhenquote, steht die Fortsetzung dieser Pillowlaven an. Die Pillows weisen gegen das Innere doleritische Gefüge auf. Der Aussenrand ist feinkörnig, besteht meist aus metamorph umgewandelten Glas (meta-hyaloklastisch) und zeigt stellenweise typische variolitische Entglasungsgefüge.

Grössere und lithologisch vielfältigere, allerdings versackte, Vulkanit-Schuppen liegen im Bachtobel südlich der Isentobel-Hütte, zwischen 1510 m.ü.M. und 1560 m.ü.M.. Neben kompakten Meta-Basalten (Relikte von in Pillowlaven eingelagerten Diabas-Sills) und variolithischen Pillows, treten auch Pillow-Brekzien und Hyaloklastite auf. Letztere weisen rosafarbenen, fein marmorisierten Calcit als Spaltenfüllungen und Matrix dieser Brekzien auf.

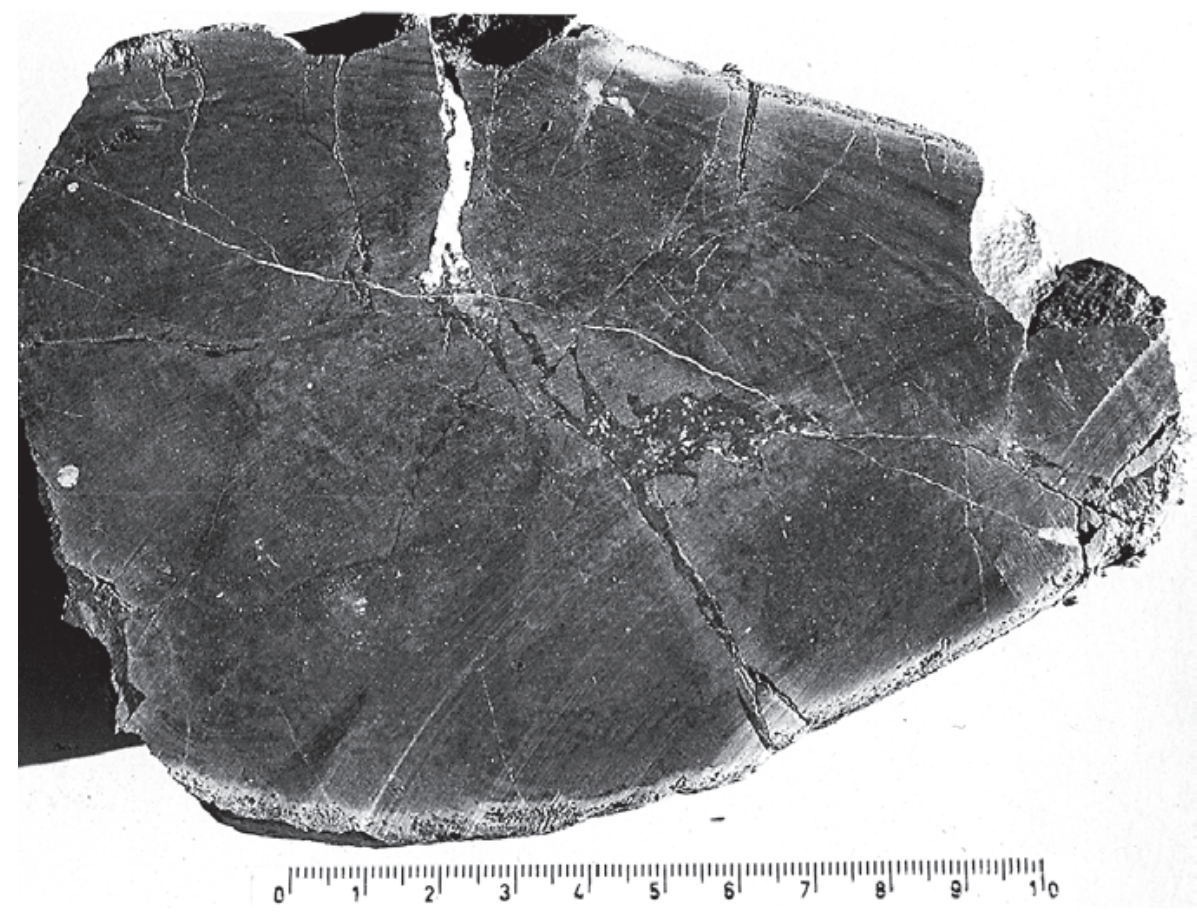

Am Roggenstock erreichen die Pillowlaven nur südlich des Ober Roggen eine Mächtigkeit von ca. 50 m (Trümpy 2006, seine Fig. 13). An einigen Stellen, namentlich bei P. 1367 im Schneiterwald sowie östlich und südlich davon, werden die Pillowlaven normal von Radiolariten des oberen Mitteljura oder des unteren Oberjura bedeckt.

\section{Petrographie}

\subsection{Pillowlaven}

Pillowlaven mit variolithischen Rändern sind meist rund bis oval und haben einen Durchmesser von ca. 0,2-1 m (Fig. 1). Sie enthalten sowohl konzentrische als auch radiale Risse und besitzen eine chloritisierte «Glas-Kruste». In den Pillows kann oft eine Änderung der Feldspatstrukturen vom Zentrum zum Rand beobachtet werden: intersertale Struktur im Zentrum, gegen aussen in intersertal-divergente, arboreszierende, sphärolitische und variolitische Strukturen (Randzone, siehe Fig. 1 \& 2) übergehend. Meistens sind diese Pillow-Laven relativ locker gepackt; die Zwischenmasse besteht dann aus Pillowbruchstücken und feinerem hyaloklastischem Material (Fig. 3), sowie aus Karbonat und rötlichem Kieselschiefer (chert).

\section{2 Hyaloklastite s.l.}

Für die Iberger Vulkanite kann, da lediglich Mineralumwandlungen stattgefunden haben und primäre Strukturen aber weitgehend erhalten blieben, die Klassifikation nicht metamorpher Hyaloklastite beibehalten werden. Unter dem Mikroskop erkennt man alle primären Formen von Gläsern mit filigranar-
Fig. 1. Hämatitisches «Mini-Pillow» mit feinem Variolithrand (siehe Analyse Ob1, Tabelle1). Mineralparagenese: Chlorit (Ripidolith), Serizit, Pumpellyit, Titanit, Hämatit, Albit, Calcit, Quarz. Lokalität: Schneiterwald Koord.: 700.53/208.78. Massstab in Zentimetern. 


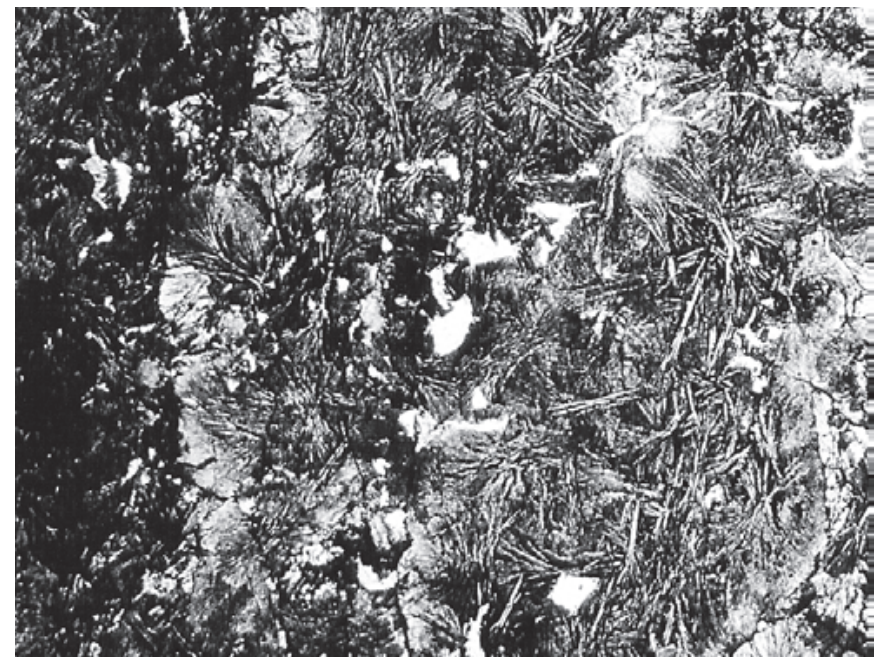

Fig. 2. Detail aus einem hyaloklastischen Pillowrand (Figur 1). Linker Bildrand: Feinster Meta-Hyaloklastit bestehend aus Chlorit, Serizit, Titanit und Hämatit; Bildmitte und rechter Rand: Die arboreszierenden Strukturen bestehen aus einem feinen Gemisch von radialstrahligem Albit und Chlorit, sowie Aktinolith oder Pumpellyit und Titanit. In den Zentren liegen MikroPhänokristalle von Albit mit unterschiedlichen Wachstumsstufen. Die dunklen Aggregate bestehen aus Chlorit, Hämatit und Titanit sowie grösseren Calcitkristallen. Bildbreite $8 \mathrm{~mm}$.

tig zonierten fluidalen Strukturen, welche meist isotrop sind und gemäss Brechungsindex und Farbe einer palagonitischen Substanz (Sideromelan-Glas und Zeolithe) nahe stehen. Röntgenographisch wird bereits ein Albit-Ripidolith-Chlorit-, respektive ein Pumpellyit-Titanit-Hämatit-Gemenge nachgewiesen. Einzelne Zonen werden durch feine Titanitsäume abgegrenzt; chloritische Zentren sind von einem feinen Titanitstaub durchsetzt.

Analoge Gesteine treten sowohl in der Arosa-Zone im Hörnli oberhalb von Arosa, als auch in den meta-basaltischen Vulkaniten der «Oberen Platta-Schuppen» im südlichen Oberhalbstein (z.B. Alp Flix) auf (Dietrich 1969).

\subsection{Diabas- und Pillowbrekzien}

Dieser heterogene Typ besteht sowohl aus Pillow-Fragmenten als auch aus diabasartigen Varietäten von Bruchstücken (Fig. 4), die meist keinen oder einen allseitigen Chloritrand besitzen. Solche Fragmente entstammen wahrscheinlich einem Lagergang (Sill) ohne vorherige Pillowbildung. Die PillowBrekzien dürften wohl durch Zerplatzen oder mechanische Aufarbeitung von Pillows entstanden sein. Die Zwischenmasse nimmt zwischen den Pillow- und Diabasfragmenten oft einen grösseren Raum ein. Von Auge erkennt man viele MiniPillows, weisse, grüne und bräunliche Fetzen umgewandelter vulkanischer Gläser (Chlorit, Albit und Pumpellyit), Variolen, Mandeln, Bläschen, sphärolitischen Calcit, grobspätigen Calcit und grosse Feldspateinsprenglinge. Bei letzteren handelt es sich um Albit mit fein verteiltem Calcit, wie röntgenographische Analysen zeigen.

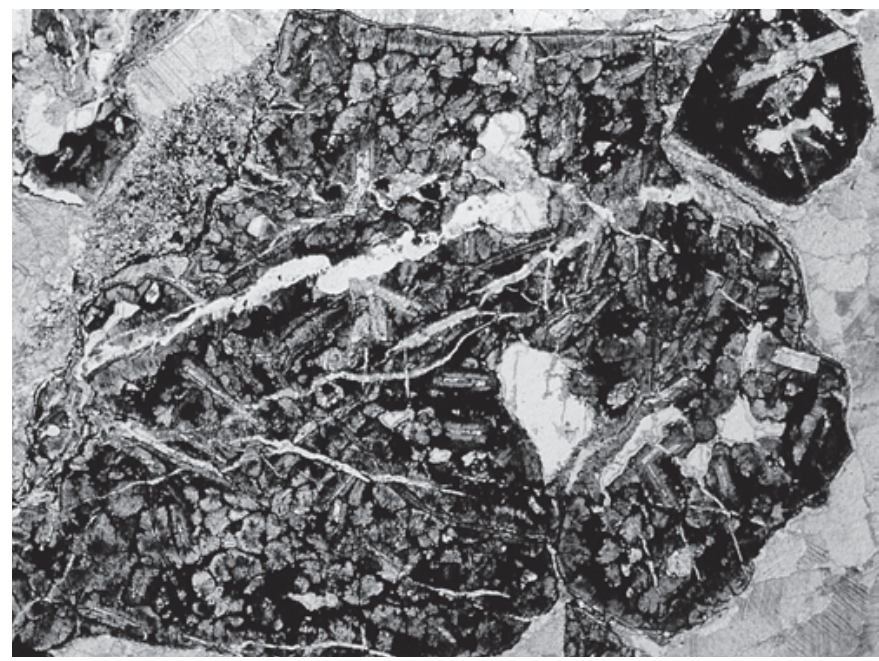

Fig. 3. Komponente aus einer Pillowbrekzie. Das Gefüge zeigt pseudomorphe Kristallaggregate aus Chlorit und Pumpellyit nach Pyroxen in einer metamorphosierten, ehemals glasigen Grundmasse (schwarz) aus feinsten Aggregaten von Hämatit, Titanit, Chlorit, Serizit, fraglichem Analzim und Quarz. Die grosse Dichte an Pyroxen Pseudomorphosen lässt als Ausgangsgestein einen Bonninit vermutet. Adern und Zwischenmasse aus Calcit, wobei der Matrixcalcit deutlich jüngeren Alters ist. Bildbreite $8 \mathrm{~mm}$. Lokalität: Schneiterwald Koord.: 700.53/208.78

Diese Vulkanit-Brekzien können sowohl in groben als auch in feinen Varietäten vorliegen. Sie sind meist an PillowLaven gebunden. Zwischen locker gepackten Pillow-Laven und den Brekzien gibt es kontinuierliche Übergänge.

\subsection{Kompakte Diabase}

Die genaue Unterscheidung zwischen Lavaerguss (flow) und lagenparallelem Gang (sill) ist in den Iberger Vulkaniten aufgrund der unzusammenhängenden Vorkommen nicht möglich. Ein konkordanter Lagergang liegt dann vor, wenn der kompakte Diabas einen relativ scharfen Kontakt zu den MetaPillow-Laven und -Brekzien aufweist. Zudem werden die Strukturen, d.h. die Grösse der Feldspat Phänokristalle gegen das Zentrum des Lagerganges gröber, d.h. von fein-intersertalen Plagioklas-Strukturen (Fig. 5) zu doleritischen Strukturen (grob inersertal bis ophitisch).

\subsection{Mineralparagenesen und metamorphe Fazies der Vulkanite}

Alle Vulkanite (Pillowlaven, -Brekzien, Hyaloklastite und kompakte Diabas-Sills) gehören gemäss ihren Mineralparagenesen der tiefsten Grünschieferfazies (Pumpellyit-Zone; Dietrich et al. 1974) an. Analzim wurde in einer Probe röntgenographisch aufgrund eines undeutlichen Spektrums vermutet, konnte aber mikroskopisch und in anderen Proben nicht nachgewiesen werden. Relikte primär magmatischer Minerale wurden nicht gefunden. Allerdings wird nicht ausgeschlossen, dass in gewissen grobkörnigeren Diabasvarietäten primäre Klinopyroxenrelikte vorliegen können. 


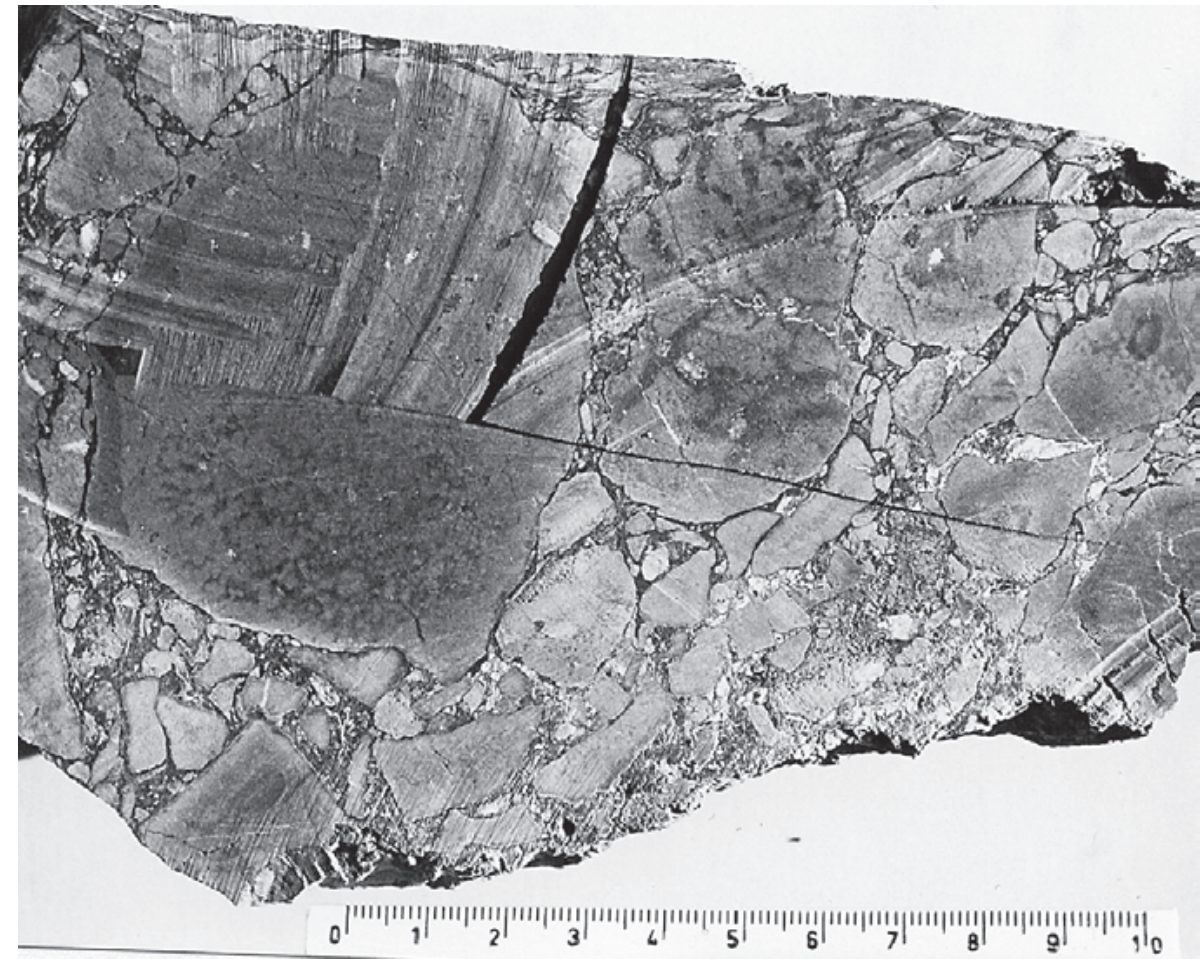

Fig. 4. Diabas- und Pillowbrekzie. Hellgrüne Diabas-Bruchstücke und Hämatit-reichere PillowFragmente (siehe $\mathrm{Ob} 6$ in Tabelle 1) liegen in einer dichten, dunkelroten Zwischenmasse aus Pumpellyit, Hämatit, Quarz, Calcit sowie wenig Chlorit und Albit. Die Diabaskomponenten zeigen intersertale bis divergente Albitgefüge und ihre Mineralparagenese ist: Chlorit (Ripidolith), Albit, Pumpellyit, Titanit und Calcit (vgl. Figur 5). Massstab in Zentimetern.

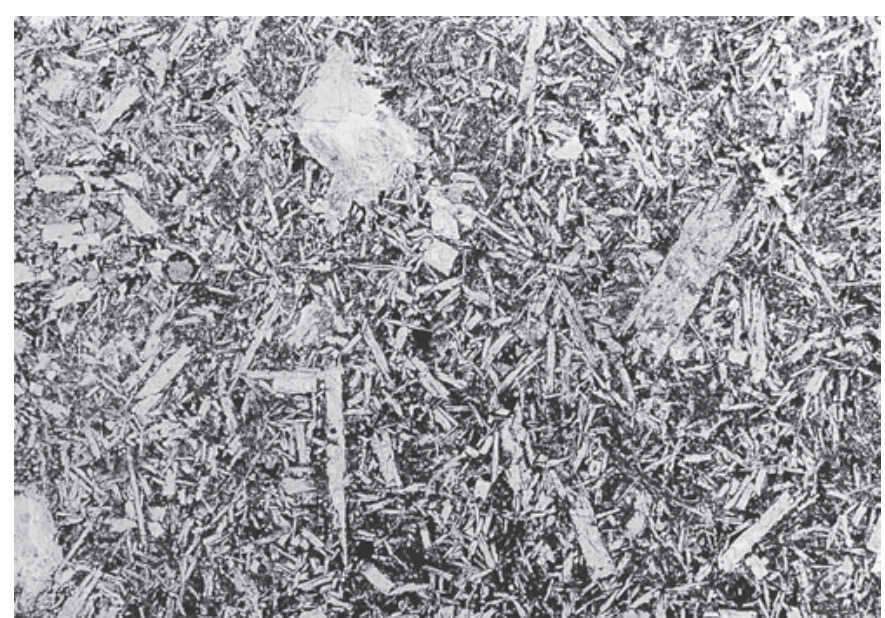

Fig. 5. Gefügebild einer Diabaskomponente (siehe Ob2 in Tabelle 1). Fein intersertales bis porphyrische Albitgefüge. Grundmasse aus Chlorit, Pumpellyit, Titanit. Bildbreite $4 \mathrm{~mm}$.

Die Mineralparagenesen der Pillowlaven bestehen aus Chlorit (Ripidolith), Serizit, Pumpellyit, Titanit, Hämatit, Albit, Calcit und Quarz. Bei den hämatitischen Varietäten nimmt in den Fragmenten der Chloritgehalt stark ab. Dafür tritt neben dem hohen Hämatitanteil zuweilen Muskovit auf. Die Meta-Basalt-Sills führen die Mineralien Chlorit (Ripidolith), Albit, Pumpellyit, Titanit und Calcit.

\section{Gesamtgesteinschemismus}

Innerhalb der Iberger Ophiolith-Schuppen (Decke) treten zwei Gruppen meta-basaltischer Gesteine auf: Pillowlaven und massive Diabase. Sechs ausgewählte chemische Gesamtgesteinsanalysen (Tabelle 1) zeigen ihre charakteristischen chemischen Eigenschaften.

\subsection{Pillowlaven}

Lediglich die Pillowlaven, Pillowbrekzien und Hyaloklastite zeigen deutliche chemische Veränderungen, welche auf Entglasungen, Lösungsprozesse und metamorphe Umwandlung ihrer Matrix und der hyaloklastischen Randzonen durch postmagmatische und metamorphe Fluide (meist $\mathrm{H}_{2} \mathrm{O}$ und $\mathrm{CO}_{2}$ ) zurückzuführen sind.

Auf wasserfreie Basis normiert variieren die $\mathrm{SiO}_{2}$ Gehalte um 50 Gew. \%, die $\mathrm{MgO}$ Gehalte zwischen 9.5 und 4.5 Gew. \%, $\mathrm{TiO}_{2}: 1.56-1.85$ Gew. \% und $\mathrm{P}_{2} \mathrm{O}_{5}: 0.17-0.21$ Gew. \%. Frische, normale MOR-Basalte zeigen tiefe Gehalte an hygro-magmatophilen Elementen wie $\mathrm{K}\left(\mathrm{K}_{2} \mathrm{O}=0.04-0.14\right.$ Gew. \%), $\mathrm{Rb}$ $(<4 \mathrm{ppm}), \mathrm{Ba}(<5 \mathrm{ppm}), \mathrm{Nb}(<3 \mathrm{ppm})$, welche während der Differentiationsprozesse stark angereichert in der fluiden Phase verteilt bleiben (Treuil \& Varet, 1973; Wood et al., 1979). Stark erhöhte $\mathrm{K}_{2} \mathrm{O}$ Gehalte, meist in submikroskopisch feinsten Serizitaggregaten, treten nur in den hyaloklastischen Pillowrandzonen sowie in der interstitiellen meta-hyaloklastischen Matrix zwischen den Pillows und den Pillowbrekzien auf. Letzteres trifft für alle hygro-magmatophile Elemente wie $\mathrm{Na}, \mathrm{Ca}, \mathrm{Sr}, \mathrm{Ba}, \mathrm{Rb}$ und der leichten Seltenen Erden zu. Die $\mathrm{Na}_{2} \mathrm{O}$ Gehalte zeigen 
Tab. 1. Gesamtgesteinschemismen der Iberger Pillowlaven und Diabase. Analysen mit Röntgenfluoreszenz (Methodik und Fehlergrenzen in Nisbet et al. 1979)

\begin{tabular}{|c|c|c|c|c|c|c|}
\hline & Ob 5 - Sill & $\begin{array}{l}\text { Ob } 2 \text { - Sill } \\
\text { Brekzie }\end{array}$ & Ob 3 - Sill & $\begin{array}{l}\text { Ob1-häm. } \\
\text { Minipillow }\end{array}$ & $\begin{array}{l}\text { Ob } 6 \text { - Pil- } \\
\text { lowkomp. }\end{array}$ & $\begin{array}{l}\text { Ob4- Pil- } \\
\text { lowrand }\end{array}$ \\
\hline \multicolumn{7}{|l|}{ Gew.\% } \\
\hline $\mathrm{SiO}_{2}$ & 44.39 & 45.50 & 48.18 & 47.14 & 47.69 & 48.55 \\
\hline $\mathrm{TiO}_{2}$ & 1.00 & 1.09 & 1.07 & 1.81 & 1.56 & 1.85 \\
\hline $\mathrm{Al}_{2} \mathrm{O}_{3}$ & 15.04 & 14.47 & 17.18 & 16.95 & 17.53 & 17.19 \\
\hline $\mathrm{Fe}_{2} \mathrm{O}_{3}$ & 2.09 & 3.06 & 2.68 & 9.50 & 6.59 & 5.98 \\
\hline $\mathrm{FeO}$ & 5.55 & 4.94 & 4.92 & 2.64 & 2.84 & 3.85 \\
\hline $\mathrm{MnO}$ & .13 & .13 & .17 & .23 & .17 & .14 \\
\hline $\mathrm{MgO}$ & 11.68 & 8.73 & 8.08 & 4.85 & 3.61 & 6.68 \\
\hline $\mathrm{CaO}$ & 9.8 & 12.50 & 8.25 & 5.95 & 9.45 & 4.37 \\
\hline $\mathrm{Na}_{2} \mathrm{O}$ & 3.08 & 3.43 & 3.76 & 5.56 & 5.23 & 5.03 \\
\hline $\mathrm{K}_{2} \mathrm{O}$ & .11 & .21 & 1.15 & .34 & .64 & 1.56 \\
\hline $\mathrm{P}_{2} \mathrm{O}_{5}$ & .09 & .12 & .10 & .17 & .17 & .21 \\
\hline $\mathrm{H}_{2} \mathrm{O}+$ & 5.35 & 5.25 & 4.00 & 5.20 & 4.20 & 4.56 \\
\hline $\mathrm{CO}_{2}$ & 2.00 & $<0.2$ & .06 & $<.02$ & .70 & $<.02$ \\
\hline Total & 100.31 & 99.43 & 99.60 & 100.34 & 100.38 & 99.98 \\
\hline $\mathrm{ppm} \mathrm{Nb}$ & 7 & 13 & 10 & 9 & 11 & 8 \\
\hline $\mathrm{Zr}$ & 75 & 86 & 77 & 127 & 121 & 144 \\
\hline $\mathrm{Y}$ & 24 & 30 & 28 & 47 & 38 & 40 \\
\hline $\mathrm{Sr}$ & 219 & 283 & 567 & 220 & 270 & 494 \\
\hline $\mathrm{Rb}$ & 5 & 11 & 23 & 13 & 21 & 48 \\
\hline $\mathrm{Pb}$ & 10 & 22 & 21 & 17 & 26 & 15 \\
\hline $\mathrm{Ga}$ & 14 & 16 & 18 & 17 & 17 & 18 \\
\hline $\mathrm{Zn}$ & 60 & 60 & 62 & 143 & 123 & 94 \\
\hline $\mathrm{Cu}$ & 49 & 54 & 96 & 53 & 67 & 27 \\
\hline $\mathrm{Ni}$ & 221 & 172 & 199 & 134 & 125 & 82 \\
\hline Co & 49 & 43 & 43 & 60 & 34 & 52 \\
\hline $\mathrm{Cr}$ & 426 & 348 & 445 & 277 & 265 & 264 \\
\hline V & 193 & 208 & 191 & 324 & 239 & 385 \\
\hline $\mathrm{Ba}$ & 36 & 48 & 190 & 46 & 52 & 242 \\
\hline Sc & 24 & 30 & 26 & 57 & 34 & 51 \\
\hline S & 312 & 172 & 176 & 182 & 175 & 152 \\
\hline
\end{tabular}

Ob 5: Tektonisierter Diabas; Strasse Schneiterwald-Isentobelhütte Koord.: 700.48/208.64

$\mathrm{Ob}$ 2: Diabaskomponente aus Diabas- und Pillowbrekzie;

Strasse Schneiterwald-Isentobelhütte Koord.: 700.48/208.64

Ob 3: Diabas (Sill); Strasse Schneiterwald-Isentobelhütte Koord.: 700.49/208.70

Ob 1: Mini-Pillow (Fig. 1); Schneiterwald Koord.: 700.53/208.78

Ob 6: Meta-Pillowlava (Fig. 4): Fragment aus Diabas- und Pillowbrekzie

Ob 4: Hyaloklastischer Rand eines Pillowfragments, Schneiterwald Koord.: 700.53/208.78

Differentiations-Abfolge (primitiv zu entwickelt): Ob5 -> Ob2 -> Ob 3; Ob4 ->Ob 1 -> Ob6 die höchsten Werte zwischen 5.03-5.56 Gew. \% (in älterer Literatur als «Spilitisierung» bezeichnet, welche im metamorphen Albit gebunden sind). Systematische Analysen aus verschiedenen Zonen vom Rand zum Zentrum einzelner Pillows aus den schwach metamorphen ophiolithischen Abfolgen im ligurischen Appenin, Mont Genèvre und Engadiner Fenster verdeutlichen die unsystematische Variation dieser Elementgehalte (Heugel, 1982). Diese scheint sowohl von den Anteilen der ursprünglichen Glasbasis zwischen den Mineralaggregaten Plagioklas, Klinopyroxen und Magnetit als auch von den Abkühlungsphänomenen (radialer und konzentrischer Rissbildung) abhängig zu sein.

Kalzium ist einerseits durch Umwandlung der ursprünglichen Plagioklase abgereichert, andererseits durch Ausfällung in Hohl- und Porenräumen als Martrix-Calcit neu gebildet worden (Fig. 3). Letzteres trifft vor allem für Pillowbrekzien und Meta-Hyaloklastite zu.

\subsection{Diabase}

Die Diabase entstammen Lagergängen zwischen den Pillowlaven. Mit Ausnahme der Hydratisierung währen der alpinen Metamorphose sind sie kaum chemisch verändert. Ihre chemische Zusammensetzung (Haupt- und Spurenelemente) gestattet deshalb eine eindeutige petrochemische Interpretation.

\section{Petrochemische und petrogenetische Interpretation}

Generell können sowohl die Pillowlaven als auch Diabase der Iberger Vulkanite als Basalte eines ehemaligen ozeanischen Rücken (analog «MORB» Mid Oceanic Ridge Basalt) gedeutet werden. Sie zeigen den typischen tholeiithischen Differentiationstrend, wobei die Diabase, insbesondere Probe Ob 5 (Tabelle 1) $\mathrm{mit} \mathrm{MgO}=11.68 \mathrm{Gew} . \%, \mathrm{Ni}=221 \mathrm{ppm}$ und $\mathrm{Cr}=426 \mathrm{ppm}$. 
Auch weisen sie tiefe Gehalte an Ti, Zr, Y, V, Sc und tiefe Werte an $\mathrm{K}_{2} \mathrm{O}$ und $\mathrm{Rb}$, also einen sehr primitiven (undifferenzierten) olivinbasaltischen Charakter, auf.

Demgegenüber sind die hämatitischen Pillowlaven mit MgO-Gehalten zwischen 6.7 und 3.5 Gew.\%, Cr zwischen 277-264 ppm und Ni 134-82 ppm, sowie mit ihren hohen Fe, Ti, $\mathrm{Zr}, \mathrm{Y}, \mathrm{V}, \mathrm{Sc}$, sowie $\mathrm{K}_{2} \mathrm{O}$ und $\mathrm{Rb}$ Gehalten hoch differenziert.

Hinweise auf unterschiedliche geochemische Eigenschaften der protolithischen basaltischen Magmen ergeben sich aus der Diskriminierung verschiedener Verhältnisse inkompatibler Spurenelemente mit einigen Haupelementen (Pearce and Cann 1973; Bickle and Pearce 1975; Pearce 1976; Shervais 1982), welche weder von Lösungsprozessen während der Diagenese noch durch die alpine Metamorphose beeinflusst wurden; so beispielsweise $\mathrm{Al}_{2} \mathrm{O}_{3} / \mathrm{TiO}_{2}$ versus $\mathrm{TiO}_{2}$, Ti/V, Ti/Zr und $\mathrm{Zr} / \mathrm{Y}$ versus $\mathrm{Zr}$ (Beccaluva et al. 1984; Bertrand et al. 1987).

Werden diese Daten mit Ergebnissen der Metabasalte der Arosa Zone und der Platta Decke (Oberhänsli und Dietrich 1975; Dürr et al. 1993; Frisch et al. 1994; Peters \& Dietrich in Vorbereitung) sowie ophiolithischen Einheiten in den Westalpen (Bertrand et al. 1987) verglichen, so zeigt sich generell eine gegenüber «normalen MORB» angereicherte geochemische Charakteristik («transitional» T-MORB: Wood et al., 1979). Die von Frisch et al. (1994) postulierte geochemische Zweiteilung zwischen Pillowbasalten und kompakten Diabasen hingegen ist sowohl auf unterschiedliche metasomatische Effekte als auch magmatische Differentiation zurückzuführen. Als Ursprung der ophiolithischen T-MOR-Basalte wird eine partielle Aufschmelzung von 10 bis $15 \%$ aus einem lherzolithischen bis harzburgitischen Mantel angenommen, möglicherweise als eine Mischung von tholeiithischen Magmen aus einem «Intra-Platten» Bereich (so genannter angereicherter «enriched E-Type MORB») mit einer Aufschmelzung der Asthenosphäre eines typischen ozeanischen Bereichs (normalen «N-Type MORB). Als Resultat ergibt sich damit ein sich langsam ausbreitender mittelozeanischer Rücken $(<2 \mathrm{~cm} / \mathrm{Jahr})$ innerhalb eines schmalen ozeanischen Becken mit mittelozeanischen Rücken, welche durch zahlreiche transforme Bruchzonen segmentiert werden. Eine derartige paleotektonische Situation wurde von zahlreichen Bearbeitern der Ophiolithsequenzen einem jurassischen Piemontesischen Ozean und seiner südwestlichen Fortsetzung in der Ligurischen Tethys zugeordnet, welches sich zwischen der Iberisch-Sardischen Mikroplatte und dem Südalpin-Apulisch-Adriatischen Mikroplattenmosaik erstreckte (Bezzi \& Piccardo 1971; Abbate et al. 1980; Conti et al. 1985; Ricou 1995).

Aufgrund umfangreicher geochemischer und isotopenchemischer vergleichender Studien der Ophiolithvorkommen zwischen Ost- und Westalpen, insbesondere der «OphiolithSchuppen» der «Col des Gêts Decke» (Bertrand 1971) sowie der Oberhalbsteiner Platta Decke modifizieren Bill et al. (2000), Demurs (2002), Schaltegger et al. (2002) und Bernoulli et al. (2003) die Interpretation eines schmalen ozeanischen Beckens insofern, als sie die paleogeographische Situation als eine Kombination zwischen dem Endstadium des Aufbrechens der Kontinente und der Exhumation kontinentaler Lithosphäre der zukünftigen Eurasischen und Afrikanischen Platten und dem Frühstadium einer Ozeanisierung («Ocean Floor Spreading») interpretieren, die während des mittleren Jura (Bajocian und Bathonian), also zwischen 170 und 160 Mio. Jahren stattfand.

\section{Ausblick}

Die Deutung plattentektonischer Prozesse aufgrund chemischer und isotopenchemischer Evidenzen, insbesondere aus unsystematisch vorkommenden, tektonisch aufgelösten rudimentären Ophiolithrelikten ist wohl indikativ aber nicht repräsentativ. Erst in Verbindung mit der Matrix (Mélange, Serpentiniten oder Flysch) der einzelnen Ophiolithglieder mit ihren assoziierten ozeanischen Sedimentanteilen sowie weiterer, meist exotischer Sedimente und Intrusiva erscheint eine tektonische und paleogeographische Interpretation möglich und glaubwürdig.

Für die Iberger Ophiolithkomponenten, zusammen mit ihren mittel- bis oberjurassischen ozeanische Sedimenten (Radiolarit und Aptychenkalk), sowie den exotischen triadischen Sedimentschuppen, können als mögliche tektonische Äquivalente sowohl die Arosa Zone als auch die «Oberen PlattaSchuppen» (Dietrich 1969 \& 1970) in Betracht gezogen werden. Es sind dies Vertreter des jurassischen «südlichen» Kontinentalrandes, der aus dem Südalpinen, Adriatischen respektive Ostalpinen Mikroplattenmosaik besteht (Dewey et al. 1973; Dietrich 1976; Weissert and Bernoulli 1985; Ricou 1995; Stampfli et al. 1998; Schmid et al. 2004).

\section{LITERATUR}

Abbate E., Bortolotti V. \& Principi P. 1980: Apennine ophiolites: a peculiar oceanic crust. In: Tethyan ophiolites 1: Western area (edited by RoccI, C.). Ofioliti Spec. Issue 59, Vol. 1 (Western Area), 59-96.

Beccaluva, L., DalPiaz, G. V. \& Maciotta, G. 1984: Transitional to normal MORB affinities in ophiolitic matabasites from the Zermatt-Saas, Combin and Antrona units, Western Alps: implications for the paleogeographic evolution of the western Tethyian basin. Geol. En Mijnbouw 63, 165-177.

Bernoulli, D., Manatschal G., Desmurs, L. \& Müntener, O. 2003: Where did Gustav Steinmann see the trinity? Back to the roots of an Alpine ophiolite concept. Geol. Soc. of America, Spec. Pap. 373, 93-110.

BERTRAND, J. 1971: Etude pétrographique des ophiolites et granites du flysch des Gèts (Haute-Savoie, France). Arch. Sci. Genève 23, 279-542.

Bertrand, J., Dietrich, V., Nievergelt, P. \& Vuagnat, M. 1987: Comparative major and trace element geochemistry of gabbroic and volcanic rock sequences, Montgenèvre ophiolite. Schweiz. Mineral. Petrogr. Mitt. 67, 147-169.

Bezzi, A. \& Piccardo, G. B. 1971: Structural features of the Ligurian ophiolites: petrologic evidence for the oceanic floor of the Northern Apennine geosyncline: A contribution to the problem of the alpine-type gabbroperidotites associations. Mem. Soc. Geologica Italiana, Roma 10, 53-63.

Bickle, M. J. \& PeArce, J. 1975: Oceanic mafic rocks in the Eastern Alps. Contrib. Mineral. Petrol. 49, 177-189.

Bill, M., NÄGler, T. \& MAsson, H. 2000: Major, minor, trace element, Sm$\mathrm{Nd}$ and $\mathrm{Sr}$ isotope compositions of mafic rocks from the earliest oceanic crust of the Alpine Tethys. Schweiz. Mineral. Petrogr. Mitt. 80, 131-145. 
Conti, M., Marcucci, M. \& Passerini, P. 1985: Radiolarian cherts and ophiolites in the northern Apennines and Corsica: age, correlations and tectonic frame of siliceous deposition. Ofiololiti 10, 203-224.

Demurs, L. 2002: Mantle evolution and magmatism in an evolving ocean-continent transition: The Platta nappe, eastern Switzerland. Ph.D. Thesis, Zürich Federal Institute of Technology, No. 14429, 143pp.

Dewey, J. F., Pitman III, W. C., Ryan W. B. F. \& Bonnin, J. 1973: Plate tectonics and the evolution of the Alpine system. Bull. geol. Soc. America 84, 3137-3180.

Dietrich, V. 1969: Die Ophiolithe des Oberhalbsteins (Graubünden) und das Ophiolithmaterial der ostschweizerischen Molasseablagerungen, ein petrographischer Vergleich.-Dissertation. Europäische Hochschulschriften, Reihe 17, Erdwissenschaften, Nr. 1, 180 S., Bern (Verlag Herbert Lang \& Cie. AG).

DiETRICH, V. 1970: Die Stratigraphie der Platta-Decke. Fazielle Zusammenhänge zwischen Oberpenninikum und Unterostalpin. Eclogae geol. Helv. 63/2, 631-671.

Dietrich, V. 1976: Evolution of the eastern Alps: A plate tectonics working hypothesis. Geology 4, 147-152.

Dietrich, V., Viagnat M. \& Bertrand, J. 1974: Alpine Metamorphism of Mafic Rocks. Schweiz. Mineral. Petrogr. Mitt. 54, 2/3, 291-332.

DÜRR, S. B., RING, U. \& FrISCH, W. 1993: Geochemistry and geodynamic significance of North Penninic ophiolites from the Central Alps. Schweiz. Mineral. Petrogr. Mitt. 73, 407-419.

Frisch, W., Ring, U., Dürr, S., Borchert, S. \& Biehler, D. 1994: The Arosa Zone and Platta Nappe Ophiolites (Eastern Swiss Alpes): Geochemical Characteristics and their Meaning for the Evolution of the Penninic Ocean. Jb. Geol. B.-A. 137, 1, 19-33.

Heugel von, W. 1982: Die Zonierung in Pillows. Ein Vergleich von frischen ozeanischen bis zu alpinen metamorphen Pillowlaven. Dissertation Phil.naturw. Fakultät Univ. Bern, 116pp.

Nisbet, E. G., Dietrich, V. J. And EsEnwein, A. 1979: Routine trace element determination in silicate minerals and rocks by X-ray fluorescence. Fortschr. Mineral. 57/2, 264-279.

Oberhänsli, R. \& Dietrich, V. 1975: Geochemische Untersuchungen an Metabasalten der alpinen Ophiolithe. Schweiz. Mineral. Petrogr. Mitt. $55 / 3,574-576$.
Pearce, J. A. (1976): Statistical analysis of of major element patterns in basalt. J. Petrol. 17, 15-43.

Pearce, J. A. And Cann, J. R. 1973: Tectonic setting of basic volcanic rocks determined using trace element analyses. Earth Planet. Sci. Lett. 19, 290-300.

Peters, TJ. \& Dietrich, V. J. in Vorbereitung: Erläuterungen, Geologischer Atlas der Schweiz 1:25 000, Blatt 1256 Bivio.

Ricou, L-E. 1995: The Plate Tectonic History of the Past Tethys Ocean. In: The Ocean Basins and Margins (eds. E.M. Nairn, B. Vrielynck, L.-E. Ricou und J. Dercourt), Vol. 8 (The Tethys Ocean), Plenum press, New York and London, 3-70.

Schaltegger, U., Desmurs, L., Manatschall, G., Müntener, O., Meier, M., Frank, M. \& Bernoulli D. 2002: The transition from rifting to seafloor spreading within a magma-poor rifted margin: field and isotopic constraints. Terra Nova 14, 156-162.

Schmid, S. M., Fügenschuh, B., Kissling, E. \& Schuster, R. 2004: Tectonic map and overall architecture of the Alpine orogen. Eclogae geol. Helv. 97/1, 93-117.

ShervaIs, J. W. 1982: Ti-V plots and the petrogenesis of modern basalts and ophiolite lavas. Earth Planet. Sci. Lett. 59, 101-118.

Stampfi, G. M., Mosar, J., Marchant, R., Masquer, D., Baudin, T. \& Borel, G. 1998: Subduction and obduction processes in the Western Alps. Tectonophysics 296, 159-204.

Treuil, M. \& VARET, J. 1973: Critères volcanologiques, pétrologiques et géochimiques de la genése et de la differenciation des magmas basaltiques: example de l'Afar. Bull. Soc. Géol. France 15, 506-540.

TRÜMPY, R. 2006: Geologie der Iberger Klippen und ihrer Flysch-Unterlage. Eclogae geol. Helv. 99, 79-121.

Weissert, H. J. \& Bernoulli, D. 1985: A transform margin in the Mesozoic Tethys. Geol. Rundschau 74, 665-679.

Wood, D. A., Joron, J.-L. \& Treuil, M. 1979: A re-apparaisal of the use of trace elements to classify and discriminate between magma series erupted in different tectonic settings. Earth Planet. Sci. Lett. 45, 326-336.

Manuskript erhalten: 24. Februar 2006

Manuskript akzeptiert: 20. März 2006

Published Online First May 17, 2006 
